\title{
Arthrogryposis-renal tubular dysfunction-cholestasis syndrome: a cause of neonatal cholestasis. Case report
}

\author{
Ozkan Ilhan, MD. ${ }^{a}$, Esra A. Ozer, Proffesor, MD. ${ }^{b}$, Senem A. Ozdemir, MD. ${ }^{c}$, Sinem Akbay, MD. ${ }^{d}$, \\ Seyma Memur, MD. ${ }^{a}$, Berat Kanar, MD. ${ }^{a}$, and Mustafa M. Tatli, Proffesor, MD.
}

\begin{abstract}
Arthrogryposis-renal dysfunction-cholestasis syndrome is a rare lethal disorder that involves multipl organ system. It is inherited autosomal recessive and caused by defects in the VPS33B and VIPAR genes. Three cardinal findings of this syndrome are arthrogryposis, renal tubular dysfunction and cholestasis. The other organ involvements including ichthyosis, central nervous system malformation, platelet anomalies, congenital heart defects and severe failure to thrive are sometimes associated with this syndrome. Clinical findings, organ biopsy and mutational analysis can help for diagnosing but there is nocurative treatment except supportive care. Several symptoms of this condition are already usually present in the neonatal period: arthrogryposis, neonatal cholestasis, skin lesions, among others. Usually survival is until the first year of life. We present a newborn whose evolution was rapidly fatal. Key words: arthrogryposis, cholestasis, proximal renal tubular dysfunction, ARC syndrome, newborn.
\end{abstract}

http://dx.doi.org/10.5546/aap.2016.eng.e9

\section{INTRODUCTION}

Arthrogryposis, renal dysfunction and cholestasis (ARC) syndrome is an autosomal recessive multisystem disorder that may be associated with germline VPS33B mutations. ${ }^{1}$ The prognosis of the syndrome is poor and most patients die by the age of 7 months because of recurrent infections, severe dehydratation, metabolic acidosis or internal hemorrhaging. ${ }^{2,3}$ We report this case since it was rapidly fatal.

a. Tepecik Training and Research Hospital. Department of Neonatology, Izmir.

b. Sitki Kocman University, Faculty of Medicine, Department of Neonatology, Mugla.

c. Dr Behcet Uz Children's Hospital.

d. Katip Celebi University, Faculty of Medicine

e. Katip Celebi University, Faculty of Medicine. Department of Neonatology, Izmir. Turkey.

E-mail Address:

Ozkan Ilhan, MD: ozkanilhan-83@hotmail.com

Funding: None.

Conflict of interest: None.

Received: 6-12-2015

Accepted: 8-3-2015

\section{CASE REPORT}

A 1-day-old female infant was admitted to neonatal intensive care unit with hypotonia, respiratory distress, abdominal distention and dysmorphic features. She was born at $38^{\text {th }}$ week of gestation from first cousin Turkish parents via cesarian section because of hydrocephaly and extremity abnormalities with a birth weight of $2000 \mathrm{~g}$. The infant was in a poor condition at birth with low Apgar scores. She had a 4 year old healthy sister. Other sibling had died after birth at 28th week of gestation because respiratory distress and dysmorphic featurs.

On admission her weight was $2000 \mathrm{~g}$ $\left(<3^{\text {th }}\right.$ percentile), the length was $38 \mathrm{~cm}\left(<3^{\text {th }}\right.$ percentile) and the head circumference was $30 \mathrm{~cm}\left(<3^{\text {th }}\right.$ percentile). Dysmorphic features included flattened nasal bridge, high arched palate, beaked nose, lax skin, micrognathia, low-set ears and microcephalia. There were generalized hypotonia, respiratory distress, hepatomegaly, abdominal distention, bell-shaped abdomen and multiple contractures such as radial deviation of the wrist, flexion contracture of the knee joints and talipes calcaneovalgus. Fracture of right femur was detected on the radiography. Ultrasonography revealed dislocation of both hip joints. Initially, the diagnosis of arthrogryposis multiplex congenita was made based on these skeletal abnormalities.

On admission to hospital hemoglobin was $18.6 \mathrm{~g} / \mathrm{dl}$, leucocytes: $19,500 / \mathrm{mm}^{3}$, platelets: $77,000 / \mathrm{mm}^{3}$ (large, pale platelets were seen in peripheral blood smear), serum total bilirubin level was $4.3 \mathrm{mg} / \mathrm{dl}$, conjugated bilirubin level $0.7 \mathrm{mg} / \mathrm{dl}$. Within 4 days, the baby developed cholestasis, dehydration due to polyuria with elevated alkaline phosphatase (ALP) with $960 \mathrm{U} / \mathrm{L}$ and normal $\gamma$-glutamyl transpeptidase (GT) with $72 \mathrm{U} / \mathrm{L}$. At age of 5 days on laboratory examination, serum total bilirubin level was $13.5 \mathrm{mg} / \mathrm{dl}$, conjugated bilirubin level $4.3 \mathrm{mg} /$ $\mathrm{dl}$, aspartate aminotransferase (AST) $555 \mathrm{U} / \mathrm{L}$ and phosphorus $2.7 \mathrm{mg} / \mathrm{dl}$. Protein and albumin values were normal. There was a prolongation of coagulation tests. Antibodies against viruses were negative. Thyroid function tests were normal. 
Plasma alpha 1 anti-tripsin, alpha feto protein, aminoacid analysis of blood and urine, tandem mass were all within normal limits. Renal tubular tests revealed elevated fractional excretion of $\mathrm{Na}$ (FENA: $4.89 \%$ ) and fractional excretion of K (FEK: $66 \%$ ), low tubuler reabsorption of phosphorus (TPR: $40 \%$ ) and proteinuria (167 mg/dL).The urine specific gravity was low (1.006). Arterial blood gases were normal. She was diagnosed as renal tubulopathy with these clinical (polyuria) and laboratory findings.

As she produced dilute urine and had polyuria, we thought nephrogenic diabetes insipidus. Abdominal ultrasound revealed bilateral mildly increased renal echogenity. Magnetic resonance cholangiopancreatography was also normal. Computed tomography showed dilatation of both lateral ventricles and third ventricle, and corpus callosum agenesis. Echocardiography revealed $2 \mathrm{~mm}$ diameter secundum ASD. As the organ biopsy including liver or kidney biopsy could be life-threatening because of tendency of bleeding, we avoided from these invasive procedures.Based on all signs and laboratory findings, the baby was diagnosed as ARC syndrome.

FiguRE 1: Dysmorphic facial features of ARC syndrome. Flattened nasal bridge, beaked nose, micrognathia, low set-ears and microcephalia

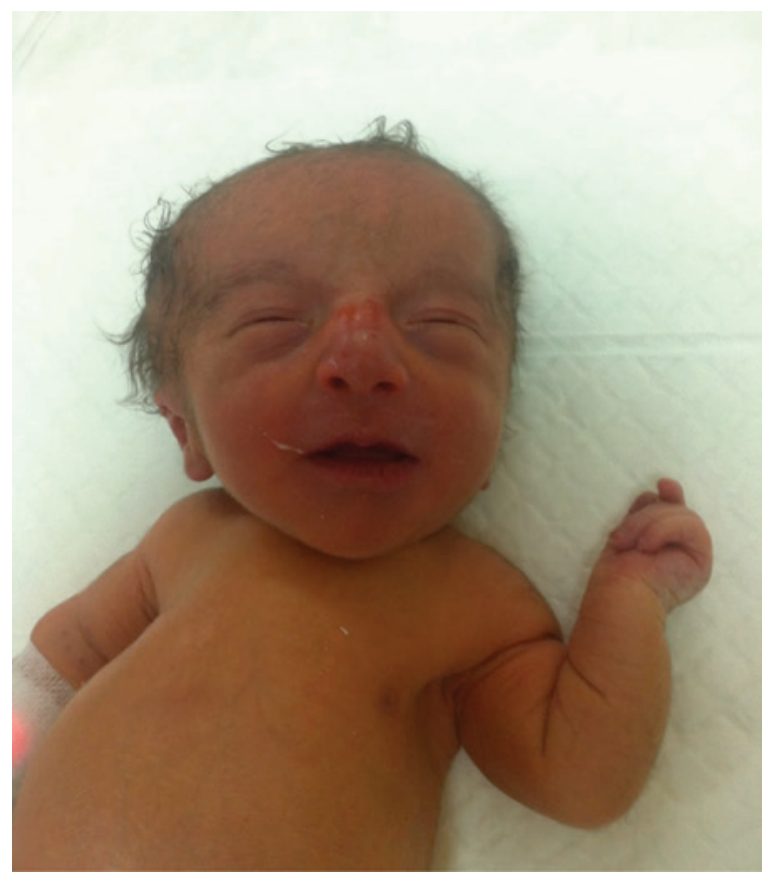

The infant could not gain weight, although she was treated with high fluid and calories via total parenteral nutrition, intravenous antibiotics, ursodeoxyhcolic acid and fat soluble vitamins. She died at the age of 37 days secondary to sepsis.

\section{DISCUSSION}

Arthrogryposis-renal dysfunction-cholestasis (ARC) syndrome is a rare autosomal recessive disease and mainly effects liver, kidney, skin, central nervous and musculoskeletal systems. Mutations in 2 interacting genes VPS33B and VIPAS39 cause this syndrome. ${ }^{2,4}$ Characteristic features of this syndrome are arthrogryposis, renal tubular dysfunction and cholestasis. ${ }^{5}$

Arthrogryposis multiplex congenita is the first diagnostic finding of ARC syndrome. ${ }^{6}$ The most common anomalies of musculoskeletal system in ARC syndrome are muscle atrophy, radial deviation of the wrist, dislocation of both hip joints, flexion contracture of the knee

FIgURE 2: Appearance of ARC syndrome. Abdominal distention, bell-shaped abdomen, lax skin, radial deviation of the wrist, flexion contracture of the knee joints and talipes calcaneovalgus

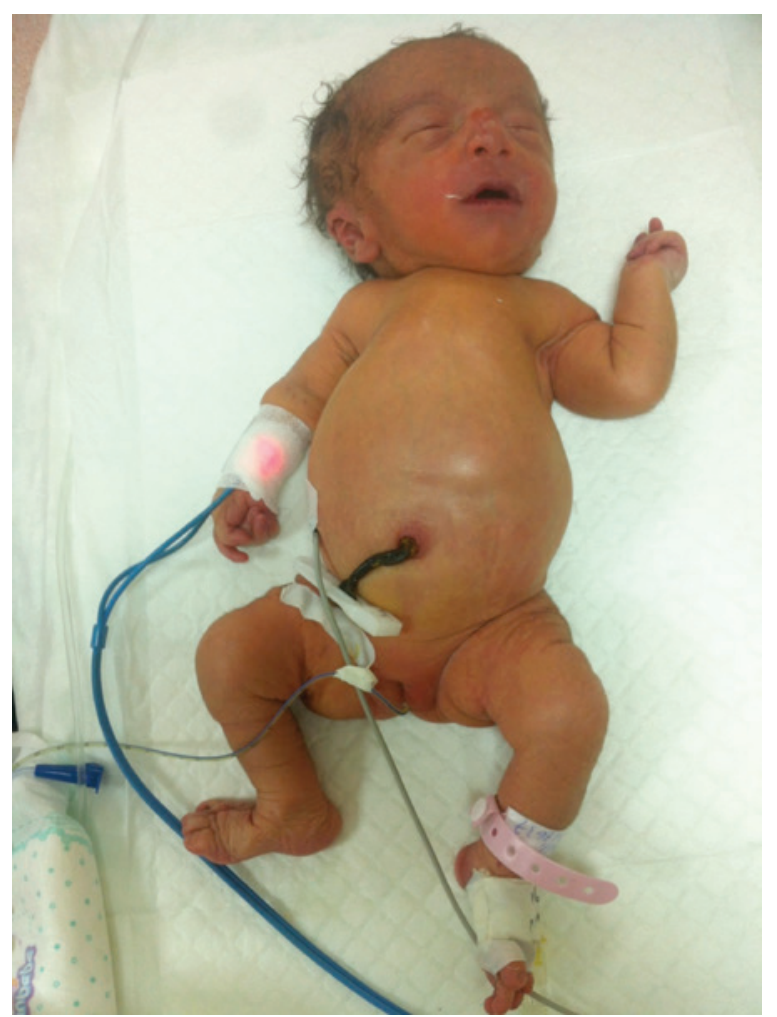


joints and calcaneovalgus. Osteopenia and pathological fractures can be observed in this syndrome owing to reduced reabsorption of phosphate ions via renal tubules and secondary to hyperparathyroidism. ${ }^{2}$ Our patient was hypotonic and had radial deviation of the wrist, flexion contracture of the knee joints and bilateral calcaneovalgus. Ultrasonography revealed dislocation of both hip joints. We thougt that fracture of the right femur was caused by osteopenia because of phosphaturia, elevated ALP values and low phosphorus levels.

Cholestatic jaundice and hepatomegaly are the most common symptoms in ARC syndrome. Low or normal GT levels, mildly elevated AST and alanine aminotransferase (ALT) levels without biliary obstruction have been described in all patients who have ARC syndrome. It's known that low GT cholestasis is one of the characteristic features of ARC syndrome. ${ }^{2,4}$ Our patient had conjugated hyperbilirubinemia with normal transaminase and GT levels as in literature. Liver histology in the patients with ARC syndrome suggests paucity of bile ducts, lipofuscin deposition and giant cell hepatitis. ${ }^{7}$ There was a prolongation of coagulation tests in our patient, so we could not perform liver biopsy due to the possibility of bleeding.

The third component of ARC syndrome is renal tubular dysfunction which is characterized by multiple features of renal Fanconi syndrome including glucosuria, phosphaturia, generalized aminoaciduria and renal tubular acidosis. Renal tubular dysfunction may present in the first few days of life or later around the age of two to three months. ${ }^{2,8,9}$ Most of the patients also present symptoms of nephrogenic diabetes insipidus.Renal ultrasonography may reveal nephrocalcinosis or a small dysplastic kidney., Our case had renal tubulopathy and nephrogenic diabetes insipidus. Renal ultrasonography showed increased of both renal echogenicity. Kim et al. ${ }^{10}$ reported 12 cases of ARC syndrome in which 10 patients had renal Fanconi syndrome and the remaining 2 patients were normal from the point of kidneys. As the renal Fanconi syndrome may be seen at the age of two to three months, we thougt that renal Fanconi syndrome did not develop yet in our patient.

Congenital heart disease and central nervous system anomalies can occasionally be seen in the patients with ARC syndrome. ${ }^{9,10}$ Our patient had $2 \mathrm{~mm}$ diameter ASD. Cerebral computed tomography showed dilatation of both lateral ventricle and third ventricle and corpus callosum agenesis in our case.

Congenital platelet abnormalities can be observed in ARC syndrome and this situation can cause bleeding tendency, therefore, platelet morphology should be examined before invasive investigations such as organ biopsy. ${ }^{9}$ Large and pale platelets were detected via peripheral blood smear in our patient.

Several dysmorphic features have been reported in this syndrome including prominent occiput, posteriorly angulated and low set-ears, flattened nasal bridge, up-slanting palpebral fissures, simian crease, high arched palate, beaked nose, lax skin and cryptorchidism. ${ }^{9}$

Failure to thrive is seen in all patients because of diarrhea and recurrent episodes of dehydratation secondary to nephrogenic diabetes insipidus. Chronic diarrhea often develops due to fat malabsorption caused by cholestasis. ${ }^{5,9}$ Our patient had failure to thrive secondary to nephrogenic diabetes insipidus, even though diarrhea was absent.

In differential diagnosis, biliary atresia was considered. The proportion of ARC to biliary atresia is around 1:7 highlighting that it may not be very uncommon in some ethnic groups, but this disorder was excluded since bile ducts were normal on abdomen ultrasonography and serum GT levels was not elevated. ${ }^{11}$ Similar clinical and laboratory findings may be seen both in ARC syndrome and progressive familial intrahepatic cholestasis (PFIC). Further genetic investigation is required to reveal the gene causing PFIC. ${ }^{6}$ Large and pale platelets with lack of $\alpha$-granules are observed both in ARC syndrome and grey platelet syndrome (GPS), whereas, GPS is generally associated with thrombocytopenia. Therefore, platelet morphology should be examined before invasive procedures. ${ }^{12}$

Unfortunately, as curative treatment for ARC syndrome is unavailable, all of the patients die within several days or months secondary to sepsis, severe dehydration and acidosis. Therefore,only supportive care including high fluid and caloric administration such as total parenteral nutrition or medium chain triglyceriderich formulas, monthly vitamin A-D-E-K and ursodeoxyhcolic acid can be given. ${ }^{2}$ Our patient died at 37 days of age because of sepsis.

Extensive research of family history, classical clinical presentations and genetic mutational analysis should be performed for diagnosing and to initiate the therapy at early stage. Once the 
patient was diagnosed genetic counseling should be provided.There were limitations of this study. We could not perform molecular studies and permission for autopsy was refused.

\section{REFERENCES}

1. Gissen P, Tee L, Johnson CA, Genin E, et al. Clinical and molecular genetic features of ARC syndrome. Hum Genet 2006;120(3):396-409.

2. Zhou Y, Zhang J. Arthrogryposis-renal dysfunction-cholestasis (ARC) syndrome: from molecular genetics to clinical features. Ital J Pediatr 2014;40:77.

3. Laforgia N, Burattini MG, Giannuzzo S, Storelli S, et al. ARC syndrome. Acta Pædiatr 2005;94(2):254-5.

4. Wang JS, Zhao J, Li LT. ARC syndrome with high GGT cholestasis caused by VPS33B mutations. World J Gastroenterol 2014;20(16):4830-4.

5. Eastham KM, McKiernan PJ, Milford DV, Ramani P, et al. ARC syndrome: an expanding range of phenotypes. Arch Dis Child 2001;85(5):415-20.

6. Tekin N, Durmu -Aydo du S, Dinleyici EC, Bör O, et al. Clinical and pathological aspects of ARC (arthrogryposis, renal dysfunction and cholestasis) syndrome in two siblings. Turk J Pediatr 2005;47(1):67-70.

7. Abdullah MA, Al-Hasnan Z, Okamoto E, Abomelha AM. Arthrogryposis, renal dysfunction and cholestasis syndrome. Saudi Med J 2000;21(3):297-9.

8. Dehghani SM, Bahador A, Nikeghbalian S, Salahi H, et al. Liver transplant in a case of arthrogryposis-renal tubular dysfunction-cholestasis syndrome with severe intractable pruritus. Exp Clin Transplant 2013;11(3):290-2.

9. Nili F, Akbari-Asbaghe P, Oloomi-Yazdi Z, Hadjizadeh N, et al. Wide spectrum of clinical features in a case of arthrogryposis-renal tubular dysfunction-cholestasis syndrome. Arch Iran Med 2008;11(5):569-72.

10. Kim SM, Chang HK, Song JW, Koh H, et al. Agranular platelets as a cardinal feature of ARC syndrome. J Pediatr Hematol Oncol 2010;32(4):253-8.

11. Ganesh R, Uppuluri R, Janakiraman L, Gowrishankar K, et al. Arthrogryposis-Renal dysfunction-Cholestasis (ARC) Syndrome: a case report. Indian J Appl Res 2015;5(3):340-1.

12. Gunay-Aygun M, Zivony-Elboum Y, Gumruk F, Geiger $\mathrm{D}$, et al. Gray platelet syndrome: natural history of a large patient cohort and locus assignment to chromosome 3p. Blood 2010;116(23):4990-5001. 